Supporting information

For

\title{
Chlorine Insertion Promoting Iron Reduction in Ba-Fe Hexagonal Perovskites: Effect on the Structural and Magnetic Properties
}

Laura Serrador ${ }^{1}$, María Hernando1, José L. Martínez², José M. González-Calbet 1,3, Aurea Varela1, F. Javier García-García3,* and Marina Parras 1,*.

1 Departamento de Química Inorgánica, Facultad de CC. Químicas, Universidad Complutense de Madrid, 28040 Madrid (SPAIN).

2 Instituto de Ciencia de Materiales, CSIC, Cantoblanco, 28049 Madrid (SPAIN).

${ }^{3}$ Centro Nacional de Microscopía Electrónica CNME, 28040 Madrid (SPAIN). 


\section{I. $10 \mathrm{H}-\mathrm{BaFeCl}_{0.13} \mathrm{O}_{3-\mathrm{y}}(0.5 \leq \mathrm{y} \leq 0.66)$}

\section{I.1. X-Ray diffraction}

Figure S1. X-ray diffraction pattern of the single phase $10 \mathrm{H}-\mathrm{BaFeCl}{ }_{0.13} \mathrm{O}_{2.48(2)}$. The le Bail profile matching is shown.

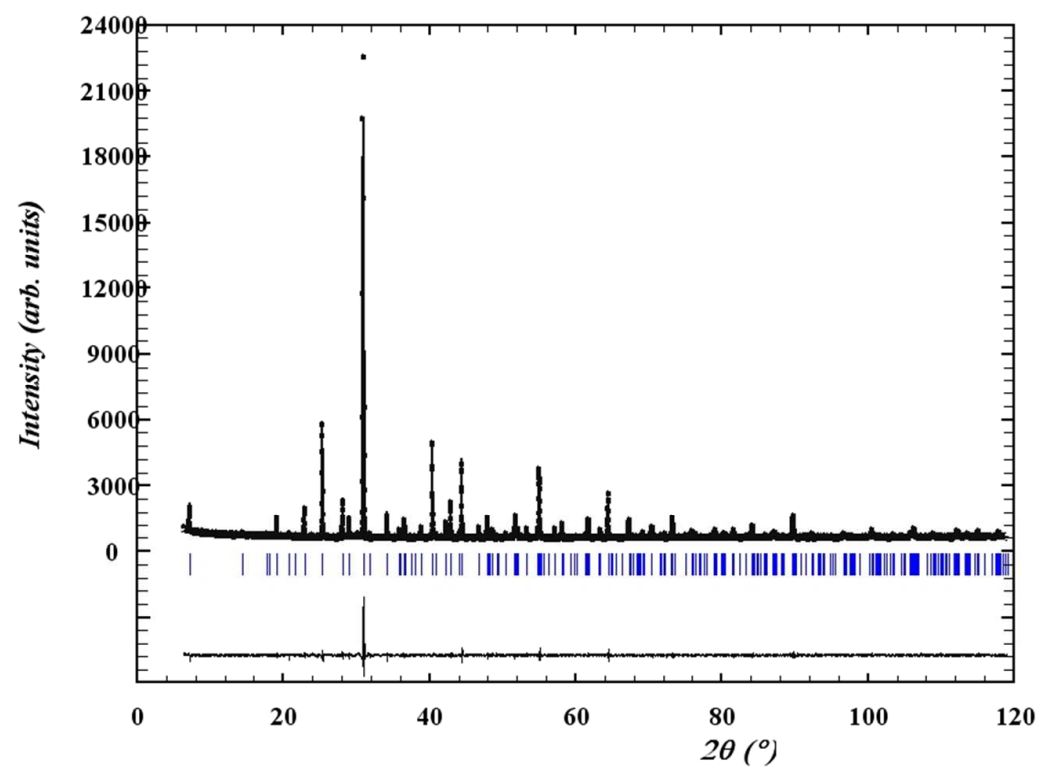

\subsection{High resolution transmission electron microscopy}

High resolution transmission electron microscopy images recorded in a crystal belonging to the $10 \mathrm{H}-\mathrm{BaFeCl}_{0.13} \mathrm{O}_{2.48(2)}$ polytype and oriented down $\langle 110\rangle$. The image in Figure $\mathrm{S} 2(\mathrm{a})$ shows a long range perfectly well ordered atomic structure in a wide area of a crystal. Results when imaging at higher magnification are presented in Figure S2(b) and (c) which correspond to the same area at different defocus conditions. The image simulations at different thickness are inset. On the right side the projected atomic potential is included to help the eye in analyzing the contrast. Note that the agreement between the experimental images and the calculated ones is excellent. Simulations were done using the refined model from neutron powder diffraction data.

The image simulation showed that the introduction of the halide into the structure, as refined from neutron powder diffraction data, does not modify the observed contrast at all. In fact, there exists no appreciable difference between images simulated by using a $10 \mathrm{H}-\mathrm{BaFeO}_{2.80}$ structure model independently of the anionic content. Therefore the analysis of the experimental contrast ends up with a basically unsolved question in what concerns the possible presence of order in the anion/vacancies sublattice. At this point the necessity to switch into the STEM mode is clear as long as this technique, with the help of a Cs corrector, might allow us to study, somehow independently, the cations and anions lattices at atomic resolution. The sub-structure cationic was imaged by setting the microscope in the STEM mode and recording the images in dark field mode. Furthermore, the collection angles used allowed us to be within the so-called "Z contrast" condition and therefore the observed contrast is directly related to the average $Z$ of the atoms. This is the High Angle Annular Dark Field mode, HAADF. This technique is perfectly well 
complemented with the parallel recording of images in the Bright Field, BF, mode. In principle, the contrast in this type of images is as complex as in conventional transmission electron microscopy. However, it is now well understood that by removing those electrons elastically scattered the anion positions can be imaged (results are presented in figures 5 (a), (b) and (c) in the manuscript).

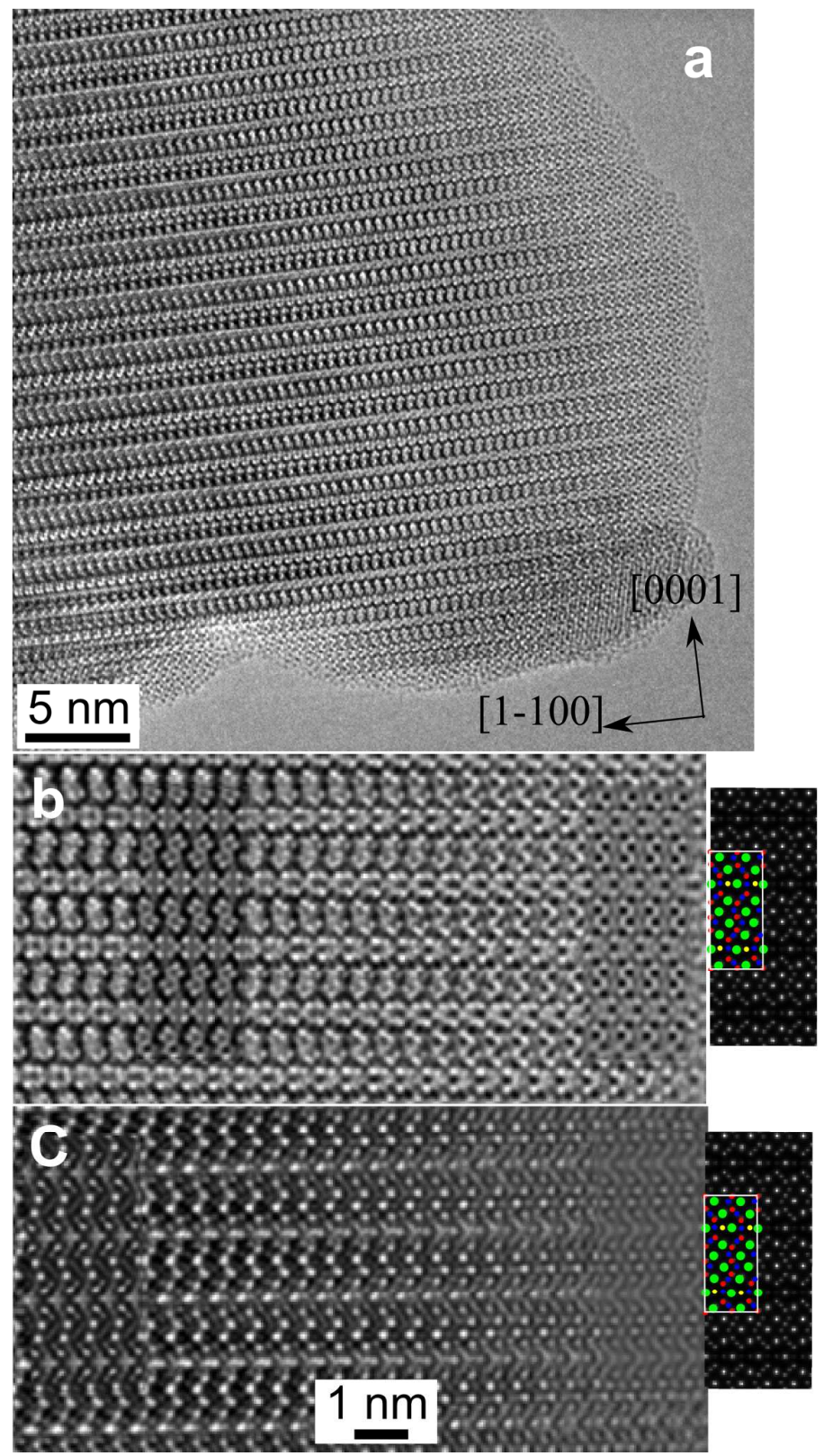

Figure S2. In (a) a conventional high resolution image of a crystal belonging to the $10 \mathrm{H}$ $\mathrm{BaFeCl}_{0.13} \mathrm{O}_{2.48(2)}$. down $\langle 110\rangle$ is presented. In (b) and (c) two magnified areas of the images are presented. The simulated images are inserted. The projected potential is shown on the right side of these two images. Green, red, blue and yellow correspond to $\mathrm{Ba}, \mathrm{Fe}, \mathrm{O}$ and $\mathrm{Cl}$, respectively. The image simulation, inset of b) and c), fits perfectly with the experimental one for b) $\Delta f=-50$ and $\Delta \mathrm{t}=8.5,2.24 \mathrm{~nm}$ and for $\mathrm{c}) \Delta \mathrm{f}=-70$ and $\Delta \mathrm{t}=8.5,2.24 \mathrm{~nm}$ and, respectively. 


\section{I.3. Thermogravimetric study}

Figure S3. (a) Thermogravimetric analysis of $10 \mathrm{H}$ - $\mathrm{BaFeCl}_{0.13} \mathrm{O}_{2.48(2)}$. sample under air from room temperature up to $900^{\circ} \mathrm{C}$. (b) X-ray diffraction patterns of the final product. All the maxima are indexed on the basis of the hexagonal $\mathrm{P}_{3} / \mathrm{mmc} 10 \mathrm{H}$ starting phase.
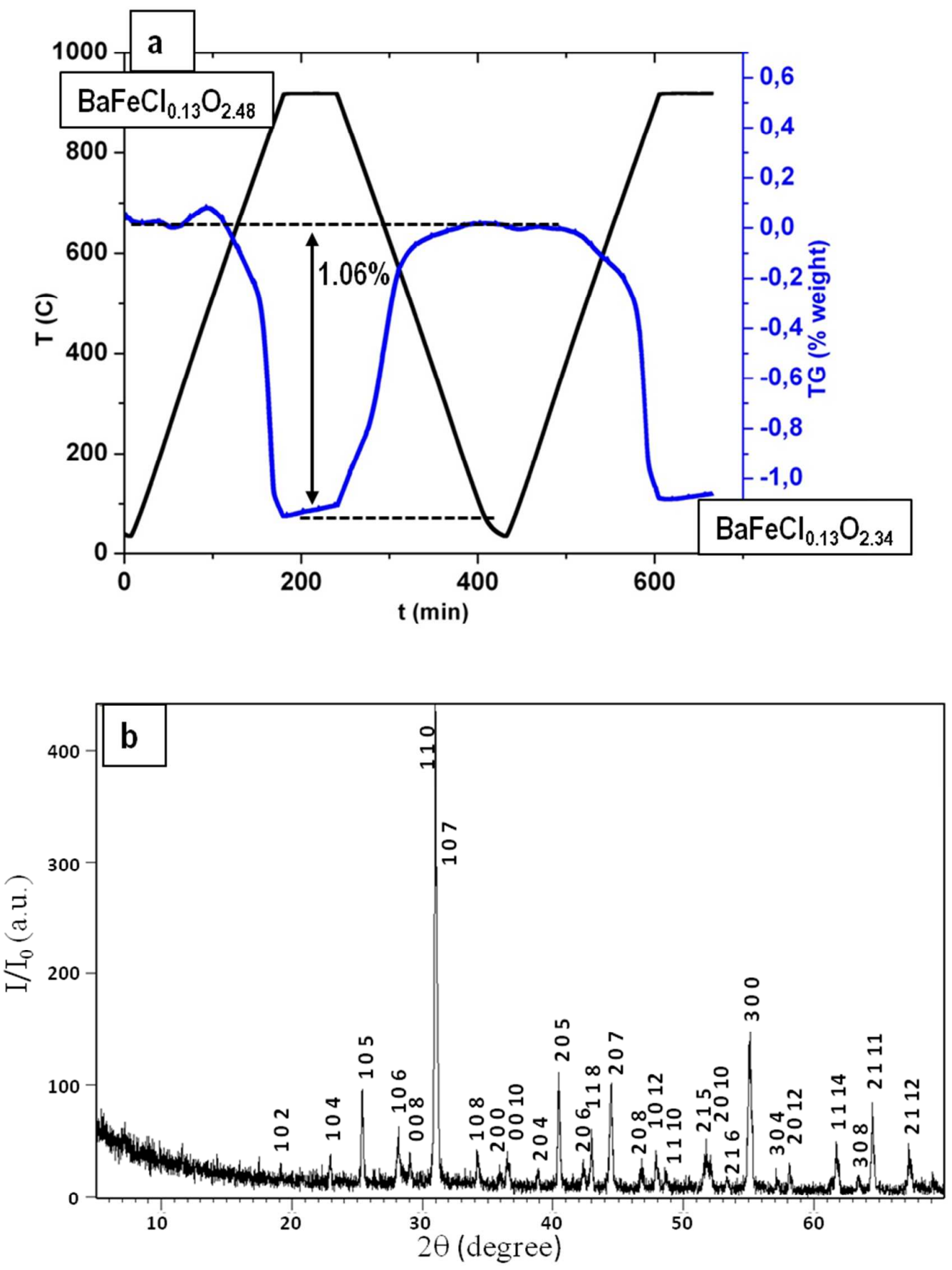


\section{II. $4 \mathrm{H}-\mathrm{BaFeCl}_{0.13} \mathrm{O}_{2.26(1)}$}

\section{II.1. Microstructural study by SAED}

The structure obtained after the high temperature transition is clearly a $4 \mathrm{H}$ type, Figure 11 . We here present some more electron diffraction information for the sake of completeness. In Figure S4 (a), (b) and (c) the $\langle 1-10\rangle,\langle 110\rangle$ and [001] electron diffraction patterns are shown. Note that the pattern in (b) is slightly tilted off to stress the gained in intensity when doing so. In (a) and (b), magnified details of the patterns are inset. In (d) the pattern recorded after tilting the crystal a few degrees away from the $<001>$ exact Bragg condition is shown.

Down the hexagonal axis, $<0001>$, there is no extra diffracted intensity but in (d) is apparent the fact that the extra diffracted intensity increases when compare to the crystal perfectly well oriented along a zone axis condition (c). Like this, we could confirm that these seemingly extra reflections are in reality just cutting point of the Ewald sphere with arcs of diffuse diffracted intensity sitting at incommensurate positions with respect to the basic reciprocal unit cell. This is clear in the fact that the extra satellite reflections are always visible when tilting while keeping excited $c^{*}$. They are clearly present at any point in between the $\langle 110\rangle$ and the $\langle 1-10\rangle$ reciprocal space directions. Note that the only possible explanation of these experimental details is the existence of the extra highly structured diffuse diffracted intensity shaped as arcs along all three presented directions simultaneously. Finally, we could confirm this hypothesis by looking at the pattern presented in Figure S4 (d). When going towards the edge of the diffraction pattern, there exit diffuse diffracted intensity shaped as spheres. These are continuous distribution of intensity that justified the explanation presented here. Furthermore, this is not the only founded peculiarity. The extra diffracted intensity follows a symmetry extinction condition in a quite similar manner as the reflections from the basic structure do. In the inset presented in (a) and (b) indexation of the extra diffracted intensity gives $F(00 / m)=0$ unless $1+m$ odd. This is indicating that the present disorder follows the same symmetry extinctions conditions that the space groups of the basic $4 \mathrm{H}$ structure itself. 


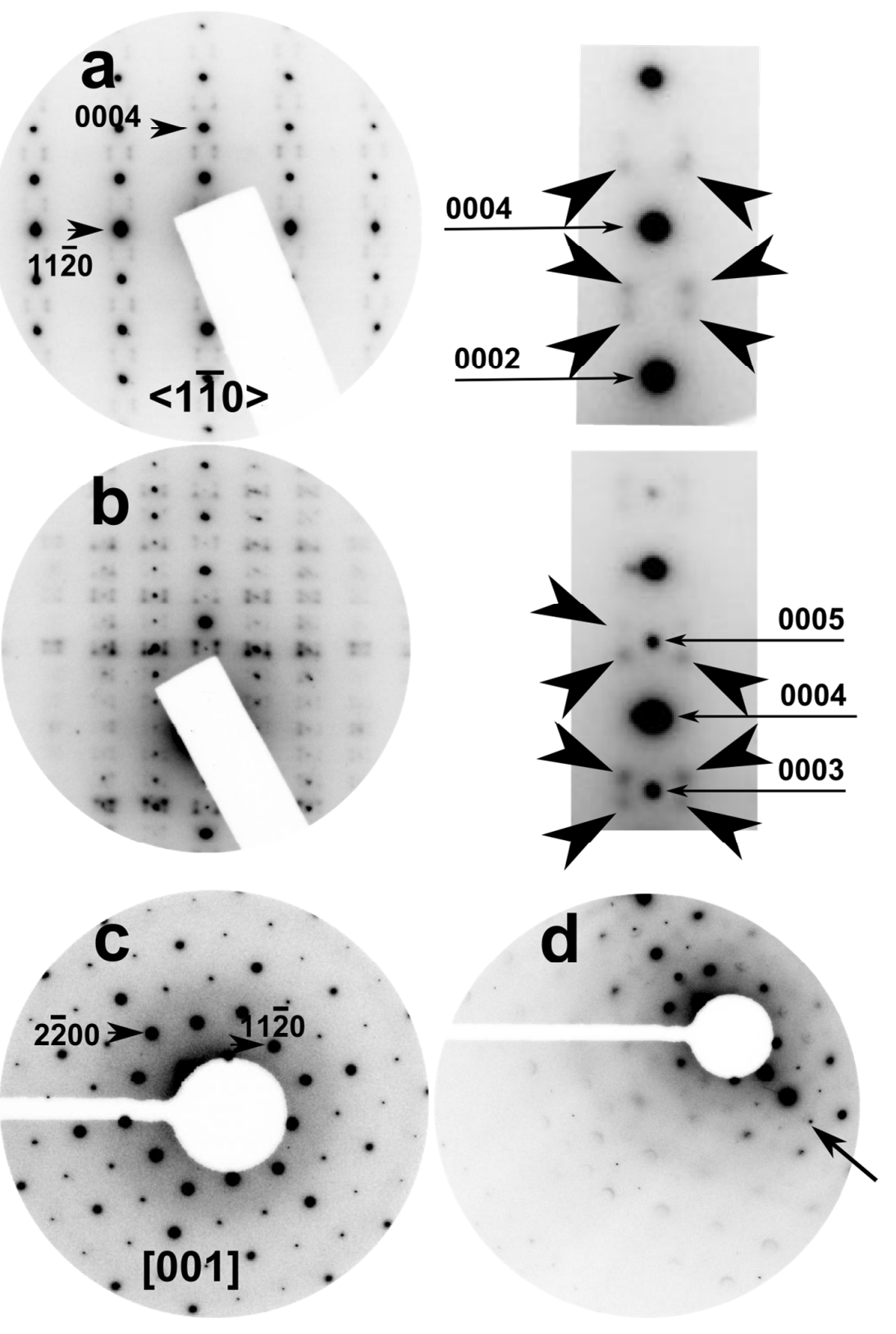

Figure S4. In (a), (b) and (c) the $\langle 1-10\rangle,\langle 110\rangle$ and [001] electron diffraction patterns are shown. Note that the pattern in (b) is slightly tilted off. In (a) and (b), magnified details of the patterns are inset on the right side of the panels. In (d) the pattern recorded after tilting the crystal a few degrees away from the $<001>$ exact Bragg condition is shown, as seen in (c). 


\section{II.2. Room temperature neutron diffraction study}

The neutron diffraction data were collected at 300K (Figure S5). For the oxyclhoride phase, the refinement was in space group $\mathrm{P} 6_{3} / \mathrm{mmc}$ using the atomic coordinates corresponding to a (hchc)$4 \mathrm{H}$ type with chlorine in $2 \mathrm{~b}$ positions. The difficulty in the refinement is increased because at room temperature is appreciable a magnetic contribution. The magnetic structure was refined using the propagation vector $k=(0,0,0)$. The 3D-AFM structure attained, represented in figure 14 of the manuscript, is similar to that found in $10 \mathrm{H}-\mathrm{BaFeCl}_{0.13} \mathrm{O}_{2.48(2)}$, ferromagnetic sheets with the magnetic moments stacked antiferromagnetically perpendicular to the c-axis. The full profile refinement for this sample also includes cubic- $\mathrm{BaFeO}_{2.5}(\mathrm{Pm}-3 \mathrm{~m})$ as additional phase. The fitting of the ND data and difference profiles between observed and calculated data is shown in Figure S5. The refined atomic positions for $4 \mathrm{H}$-structure resulting from the refinement are listed in Table S1 and selected interatomic distances are gathered in table S2.

According to this refinement, $\mathrm{BaFeCl}_{0.13(1)} \mathrm{O}_{2.26(1)}$ composition per unit formula is obtained showing that the chlorine content is conserved in the $10 \mathrm{H} \rightarrow 4 \mathrm{H}$ structural transformation according to the compositional EDS result. The anionic content of the hexagonal layers results $\left(\mathrm{BaCl}_{0.26(1)} \mathrm{O}_{1.53(1)}\right)$ that should corresponds to a mixture close to $50 \%$ of tetrahedral and $50 \%$ octahedral environment for $\mathrm{Fe}$ in the hexagonal slabs.

As a consequence, the structure results highly disordered as evidence by the splitting and/or of anomalous values of isotropic thermal factors of atoms. For example, in a first attempt, an isotropic thermal factor of $5.9 \AA^{2}$ was attained for $\mathrm{Ba} 1$ atoms. A displacement of this cation shutting up and down along $\mathrm{C}$-axis $(\mathrm{z} \pm 0.036)$ was considered in agreement with the contrast observed in the TEM image (figure 13 in the manuscript). This result is shown in table S2. This displacement of $\mathrm{Ba} 2$ atoms could be associated to the incorporation of the chlorine atoms in the adjacent hexagonal layers (as previously observed in the 10H-phase). The structural disorder in the hexagonal layers is also reflected in the high isotropic thermal factor values for $\mathrm{O} 2$ atoms $\left(2.09 \AA^{2}\right)$

Figure S5. Rietveld refinement of Neutron diffraction data at room temperature of $4 \mathrm{H}$ $\mathrm{BaFeCl}_{0.13} \mathrm{O}_{2.26}$. The solid lines and overlying dots indicate the calculated and observed intensities. The second phase corresponds to the antiferromagnetic magnetic structure which exists at roomtemperature.

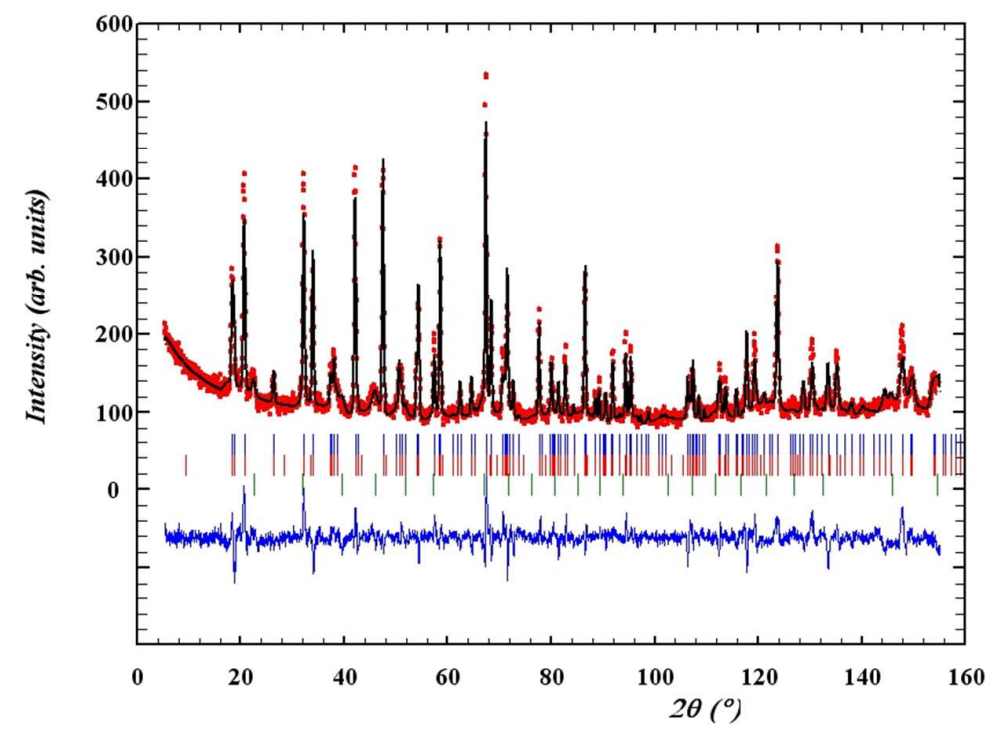


Table S1. Refined atomic positions for $4 \mathrm{H}$-structure.

\begin{tabular}{|l|c|c|c|c|c|}
\hline & $\mathbf{x} / \mathbf{a}$ & $\mathbf{y} / \mathbf{b}$ & $\mathbf{z} / \mathbf{c}$ & $\mathbf{B i s o}\left(\AA^{2}\right)$ & Occ \\
\hline $\mathrm{Ba} 1 \mathrm{a}$ & 0 & 0 & $+0.0360(8)$ & $1.09(19)$ & 0.5 \\
\hline $\mathrm{Ba} 1 \mathrm{~b}$ & 0 & 0 & $-0.0360(8)$ & $1.09(19)$ & 0.5 \\
\hline $\mathrm{Ba} 2$ & $1 / 3$ & $2 / 3$ & 0.25 & $0.82(11)$ & 1.0 \\
\hline $\mathrm{Fe} \mu=2.06(7) \mathrm{M}_{\mathrm{B}}$ & $1 / 3$ & $2 / 3$ & $0.600(3)$ & $1.09(4)$ & 1.0 \\
\hline $\mathrm{Cl}$ & 0 & 0 & 0.25 & $0.71(2)$ & $0.26(1)$ \\
\hline $\mathrm{O} 1$ & 0.5 & 0 & 0 & $1.69(2)$ & 1.0 \\
\hline $\mathrm{O} 2$ & -0.18907 & -0.37816 & 0.25 & $2.09(2)$ & $0.51(1)$ \\
\hline
\end{tabular}

$a=5.74511(8) \AA, c=9.7754(2) \AA$

$\mathrm{RB}=12.2, \mathrm{X}^{2}=3.74$.

Table S2. Selected interatomic distances

\begin{tabular}{|c|c|}
\hline Atoms & $d(\AA)$ \\
\hline Ba1-01 x 6 & $2.8949(1)$ \\
\hline $\mathrm{Ba} 1-02 \times 3$ & $2.808(7)$ \\
\hline $\mathrm{Ba} 1-\mathrm{O} 2 \times 3$ & $3.376(8)$ \\
\hline Ba2-01 x 6 & $2.9535(3)$ \\
\hline $\mathrm{Ba} 2-\mathrm{O} 2 \times 6$ & $2.881(7)$ \\
\hline $\mathrm{Fe}-01 \times 3$ & $1.9251(15)$ \\
\hline $\mathrm{Fe}-\mathrm{O} 2 \times 3$ & $2.051(4)$ \\
\hline Cl-Ba1a x 2 & $2.0858(8)$ \\
\hline Cl-Ba1a x 2 & $2.802(8)$ \\
\hline Cl-Ba1b $\times 2$ & $2.0858(8)$ \\
\hline Cl-Ba1b $\times 2$ & $2.802(8)$ \\
\hline
\end{tabular}




\section{II.3. Magnetic Properties.}

Figure S6. Magnetic susceptibility versus temperature of $4 \mathrm{H}-\mathrm{BaFeCl}_{0.13} \mathrm{O}_{2.26}$ under an applied field of $500 \mathrm{Oe}$.

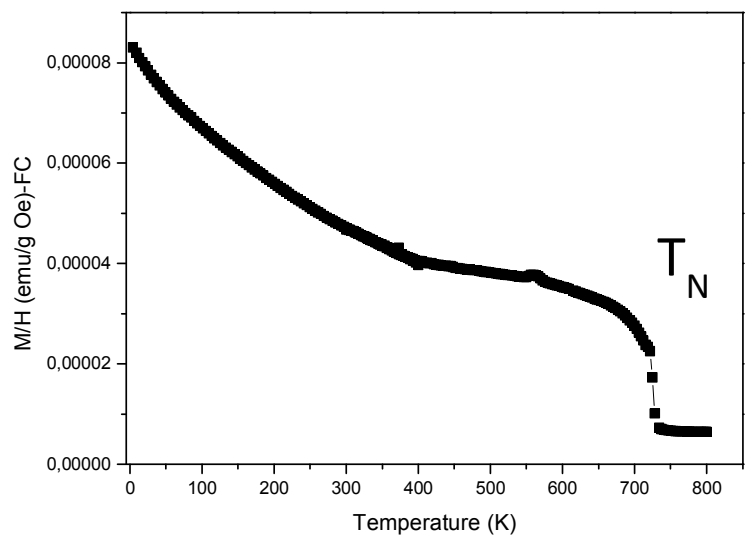

The dependence of the magnetization with temperature reveals a similar behaviour than that described for the $10 \mathrm{H}-\mathrm{BaFeCl}_{0.13} \mathrm{O}_{2.48(2)}$ in the manuscript. The increase of the susceptibility at temperature close to $730 \mathrm{~K}$ could be associated to the Neel temperature, reflecting the strong AFM exchange interactions Fe-O-Fe at $180^{\circ}$. 